\title{
CONTRIBUCIÓN AL ESTUDIO POLÍNICO DE ESPECIES ORNAMENTALES: ACANTHACEAE Y VERBENACEAE
}

\author{
M. Mar TRIGO
}

\begin{abstract}
RESUMEN. Contribución al estudio polínico de especies ornamentales: Acanthaceae y Verbenaceae. Se estudian con el microscopio óptico (MO) y con el microscopio electrónico de barrido (MEB) la morfología polínica de 14 especies pertenecientes a las familias Acanthaceae (8) y Verbenaceae (6) habitualmente cultivadas como ornamentales en el sur de España.

Mientras que las especies estudiadas de Verbenaceae presentan tipos polínicos similares, trizonocolporados o trizonocolpados de superficie psilado-perforada o perforado-reticulada, encontramos más variedad de tipos polínicos en Acanthaceae que van desde el trizonocolpado y trizonocolporado al heterocolpado, bizonocolporado y espiraperturado, con ornamentaciones muy diversas.
\end{abstract}

Palabras clave. Polen, Acanthaceae, Verbenaceae, ornamental, España.

ABSTRACT. Contribution to the palynological study on ornamental species: Acanthaceae and Verbenaceae. The pollen morphology of 14 species of Acanthaceae (8) and Verbenaceae (6) usually cultivated in southern Europe, has been studied by light and scanning electron microscopy. Pollen was acetoysed by the method of Erdtman (1960) modificated by Reitsma (1969) and mounted in glycerine jelly for light microscopy. For scanning electron microscopy the pollen were coated of gold evaporate.

The taxa examined of Verbenaceae has 3-colporate or 3-colpate pollen grains with psilate-perforate or perforate-reticulate surface. Instead, Acanthaceae present trizonocolpate, trizonocolporate, heterocolpate, bizonocolporate and spiraperturate pollen types with various ornamentation.

Key words. Pollen, Acanthaceae, Verbenaceae, ornamental, Spain.

\section{INTRODUCCIÓN}

La familia Acanthaceae agrupa alrededor de 250 géneros y unas 2.500 especies distribuidas fundamentalmente por las regiones tropicales de todo el Mundo, aunque con algunos representantes en las zonas templadas. Algo similar ocurre con la familia Verbenaceae que, aunque también cuenta con algunas especies propias de zonas templadas, la inmensa mayoría de sus alrededor de 75 géneros y más de 3.000 especies, presentan distribución eminentemente tropical y subtropical.

Muchas de estas especies poseen inflorescencias grandes y vistosas que han facilitado su difusión como plantas ornamentales en los jardines de clima cálido y subtropical, habiéndose observado que sus flo- 
res son visitadas repetidamente por insectos, fundamentalmente hormigas y abejas, atraidas por el abundante néctar que segregan (especialmente Acanthaceae) y por la viva coloración de la corola, siendo probable que el polen de las mismas aparezca en el contenido de mieles procedentes de colmenas próximas a núcleos urbanizados como así ha sido recogido por Belmonte et al. (1986).

El presente trabajo pretende ser una continuación de otros ya publicados anteriormente (Trigo, 1989; Trigo y García, 1990; Trigo, 1991, 1992a, 1992b) como contribución al estudio polínico de plantas cultivadas.

\section{MATERIAL Y MÉTODOS}

Para el estudio polínico se ha utilizado tanto material fresco, recién recolectado e incluido en ácido acético glacial, como material seco procedente del Herbario del Departamento de Biología Vegetal de la Facultad de Ciencias de la Universidad de Málaga (MGC). En todos los casos dicho material se trató siguiendo el método acetolítico de Erdtman (1960) modificado por Reitsma (1969). El montaje para su observación con el microscopio óptico se realizó usándose como medio glicerogelatina incolora y sellándose posteriormente las preparaciones con parafina. Para su observación con el microscopio electrónico de barrido (MEB) se recubrieron las muestras con oro vaporizado en atmósfera de alto vacío, apareciendo en cada una de las microfotografías obtenidas las siguientes inscripciones, de izquierda a derecha: número de archivo de la foto, kilovoltaje al que fue sometida la muestra, microescala y distancia de trabajo (WD), expresada en milímetros.

Para calcular las dimensiones de los ejes polar (P), ecuatorial (E) y diámetro (D), en su caso, se han efectuado 30 medidas por muestra y para los demás caracteres al menos 15 observaciones. Los valores que aparecen entre pa- réntesis corresponden a la media aritmética y a la desviación típica del intervalo.

La terminología empleada en las descripciones es, fundamentalmente, la propuesta por Erdtman (1945, 1952), Faegri \& Iversen (1975), Hideux \& Ferguson (1975), Reitsma (1970), Van Campo (1958) y Wodehouse (1935).

En el texto, las especies aparecen, dentro de cada familia, ordenadas alfabéticamente, habiéndose agrupado aquéllas cuyos granos de polen presentan una morfología similar. Se incluye, además, el areal natural de distribución de las mismas así como las referencias que hemos encontrados sobre estudios anteriores.

\section{RESULTADOS}

\section{ACANTHACEAE}

Acanthus mollis L. (Región Mediterránea).

Polen trizonocolpado. Isopolar, radiosimétrico. Triangular en v.p. y c.o.e., planaperturado; elíptico obtuso en v.e. y c.o.m., de prolado a perprolado, siendo $\mathrm{P} / \mathrm{E}=1,83$ $2,30(2 \pm 0,14)$. Tamaño grande, con valores de $\mathrm{P}=52-60(56,24 \pm 2,06) \mu \mathrm{m}$ y de $\mathrm{E}=26-32$ $(28,16 \pm 1,65) \mu \mathrm{m}$. Aberturas simples; de tipo colpo, muy estrechos y con la membrana apertural difícil de apreciar. Exina de unos $3 \mu \mathrm{m}$ de grosor en los polos, llegando hasta los $5 \mu \mathrm{m}$ en la mesocolpia debido a un engrosamiento de la sexina, siendo ésta aproximadamente 1,5 veces más gruesa que la nexina en los polos y de 3 a 4 veces en la mesocolpia. Téctum parcial. Infratéctum columelado. Superficie regularmente reticulada, con lúmenes redondeados de hasta $0,8 \mu \mathrm{m}$ de diámetro y provistos de un gránulo en su parte central; muros truncados (fig. 1).

Referencias: Luque y Candau (1987a), Roure (1985).

Eranthemum nervosum R. Br. (India).

Polen trizonocolporado. Isopolar, 

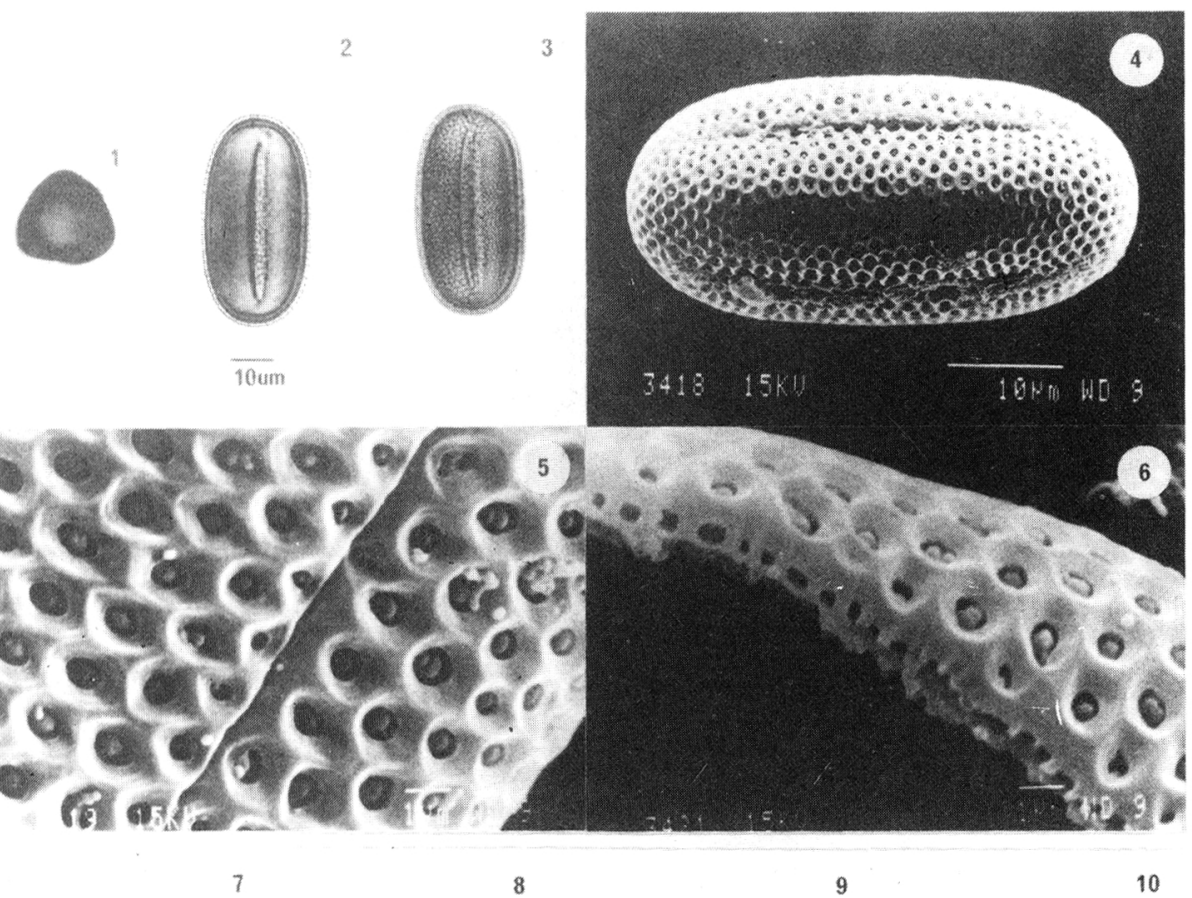

9
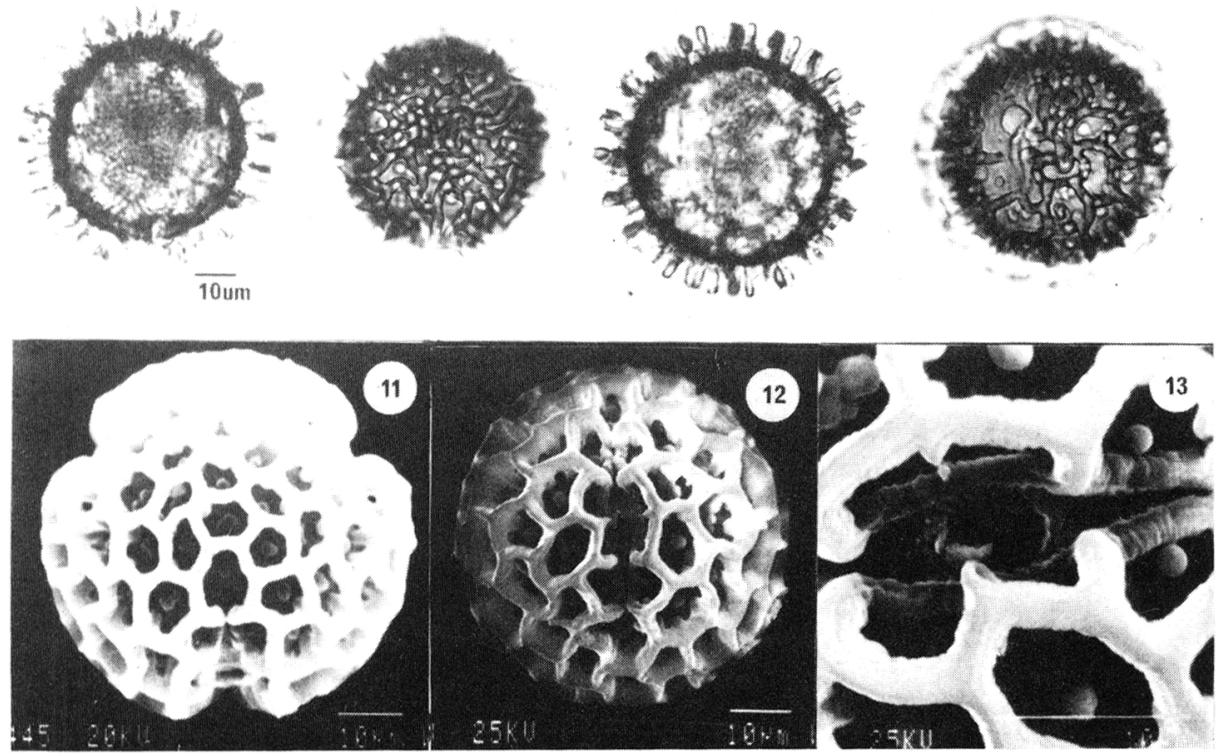

Figura 1. Acanthus mollis: 1) v.p. y c.o.e. 2) v. e. y c.o.m. 3) v. e., superficie y abertura. 4) Aspecto general del polen, v.e. 5) Detalle de la superficie y abertura 6) Detalle de la estratificación de la exina. Eranthemun nervosum: 7) v.p. y c.o.e. 8) v.p., superficie. 9) v.e. y c.o.m. 10) v.e., superficie y abertura. 11) Aspecto general del polen, v. p. 12) Aspecto general del polen, v.e. 13) Detalle de la superficie y abertura. 
radiosimétrico. Circular en v.p. y c.o.e; circular en v.e. y c.o.m., de oblado-esferoidal a prolado-esferoidal, siendo $\mathrm{P} / \mathrm{E}=0,91-1,08$ $(0,99 \pm 0,03)$. Tamaño grande, con valores de $\mathrm{P}=62-75(67,23 \pm 3,86) \mu \mathrm{m}$ y de $\mathrm{E}=63-75$ $(68,23 \pm 3,61) \mu \mathrm{m}$. Aberturas compuestas; ectoaberturas de tipo colpo, subterminales, bordeadas de un estrecho margo de unos $2 \mu \mathrm{m}$ de anchura y con la membrana apertural escábrida. Exina de 11 a $13 \mu \mathrm{m}$ de grosor, con la sexina unas 3 veces más gruesa que la nexina. Téctum parcial. Infratéctum columelado, con columelas gruesas y ensanchadas en la parte apical. Superficie reticulada, con lúmenes irregulares de hasta $15 \mu \mathrm{m}$ de longitud y muros ondulados, psilados, apareciendo columelas libres en el interior de los lúmenes (fig. 1).

Referencias: Mateu et al. (1987).

Hypoestes sanguinolenta (Van Houtte) Hook. (Madagascar)

Jacobinia suberecta E. André (Uruguay).

Polen 9-heterocolpado. Isopolar, radiosimétrico. Triangular en v.p. y c.o.e., planaperturado; elíptico en v.e. y c.o.m. De prolado a perprolado, siendo $\mathrm{P} / \mathrm{E}=1,50-1,87$ $(1,67 \pm 0,12)$ en $H$. sanguinolenta y $1,49-2,33$ $(1,80 \pm 0,16)$ en $J$. suberecta. Tamaño de mediano a grande, con valores de $\mathrm{P}=41-50$ $(46,75 \pm 2,84) \mu \mathrm{m}$ en $H$. sanguinolenta y 63-82 $(71,8 \pm 4,6) \mu \mathrm{m}$ en $J$. suberecta y de $\mathrm{E}=26-31$ $(28,05 \pm 1,70) \mu \mathrm{m}$ en $H$. sanguinolenta y $33-44$ $(39,44 \pm 2,39) \mu \mathrm{m}$ en $J$. suberecta. Sistema apertural compuesto por 9 aberturas, 6 de ellas colpadas y otras 3 colporadas, paralelamente dispuestas al eje polar en 3 grupos de 3 , de tal forma que cada una de las aberturas colporadas queda flanqueada por sendas aberturas colpadas, probablemente éstas últimas con función harmomegata. Ectoaberturas de tipo colpo, terminales, con la membrana apertural microrreticulada en $H$. sanguinolenta y granulosa en $J$. suberecta; endoaberturas de tipo poro, lalongadas, ligeramente elípticas, de $4-5 \times 6-7 \mu \mathrm{m}$. Exina de $3-4 \mu \mathrm{m}$ de grosor en los polos y de 5 a $6 \mu \mathrm{m}$ en la mesocolpia, siendo la sexina unas 2 veces más gruesa que la nexina, engrosándose ésta última a nivel de las endoaberturas para formar costillas. Téctum parcial. Infratéctum columelado. Superficie reticulada, con lúmenes de hasta $1,5 \mu \mathrm{m}$ de diámetro de borde ondulado en $J$. suberecta, que dejan ver las columelas del infratéctum; muros psilados (fig. 2).

Referencias: se desconocen.

Justicia adhatoda L. (India y Sri Lanka)

Polen bizonocolporado. Isopolar, bisimétrico. Elíptico en v.p. y c.o.e., elíptico obtuso en v.e. y c.o.m. Prolado, siendo $\mathrm{P} / \mathrm{E}_{1}=$

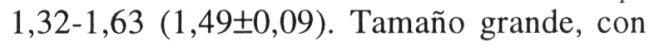
valores de $\mathrm{P}=50-66(56,95 \pm 4,06) \mu \mathrm{m}$, de $\mathrm{E}_{1}=$ $35-41(38 \pm 2,11) \mu \mathrm{m}$ y de $E_{2}=25-31$ $(28 \pm 2,39) \mu \mathrm{m}$. Aberturas compuestas; ectoaberturas de tipo colpo, subterminales, con los extremos agudos, de 25-32 $\mu \mathrm{m}$ de longitud, y con la menbrana apertural granulosa; endoaberturas de tipo poro, generalmente lalongadas, a veces circulares, de 2,5-5 x 3$6,5 \mu \mathrm{m}$. Cada abertura se encuentra flanqueda por dos zonas deprimidas del téctum, a modo de pseudocolpos, sobre los que se disponen en forma de ínsulas porciones más elevadas del mismo, en número de 5 a 8 , de contorno circular o ligeramente elíptico de hasta $5 \mu \mathrm{m}$ de diámetro y de ornamentación similar a la mesocolpia. Exina de aproximadamente $3 \mu \mathrm{m}$ de grosor en los polos y de 4 a $5 \mu \mathrm{m}$ en el ecuador, con la sexina mucho más gruesa que la nexina, engrosándose ésta última a nivel de las endoaberturas para formar costillas. Téctum parcial. Infratéctum columelado. Superficie finamente reticulada, con lúmenes de hasta $0,5 \mu \mathrm{m}$ de diámetro y con las elevaciones y depresiones del téctum antes mencionadas (fig. 3).

Referencias: Mateu et al. (1987).

Justicia carnea Hook. (Brasil)

Polen bizonocolporado. Isopolar, 

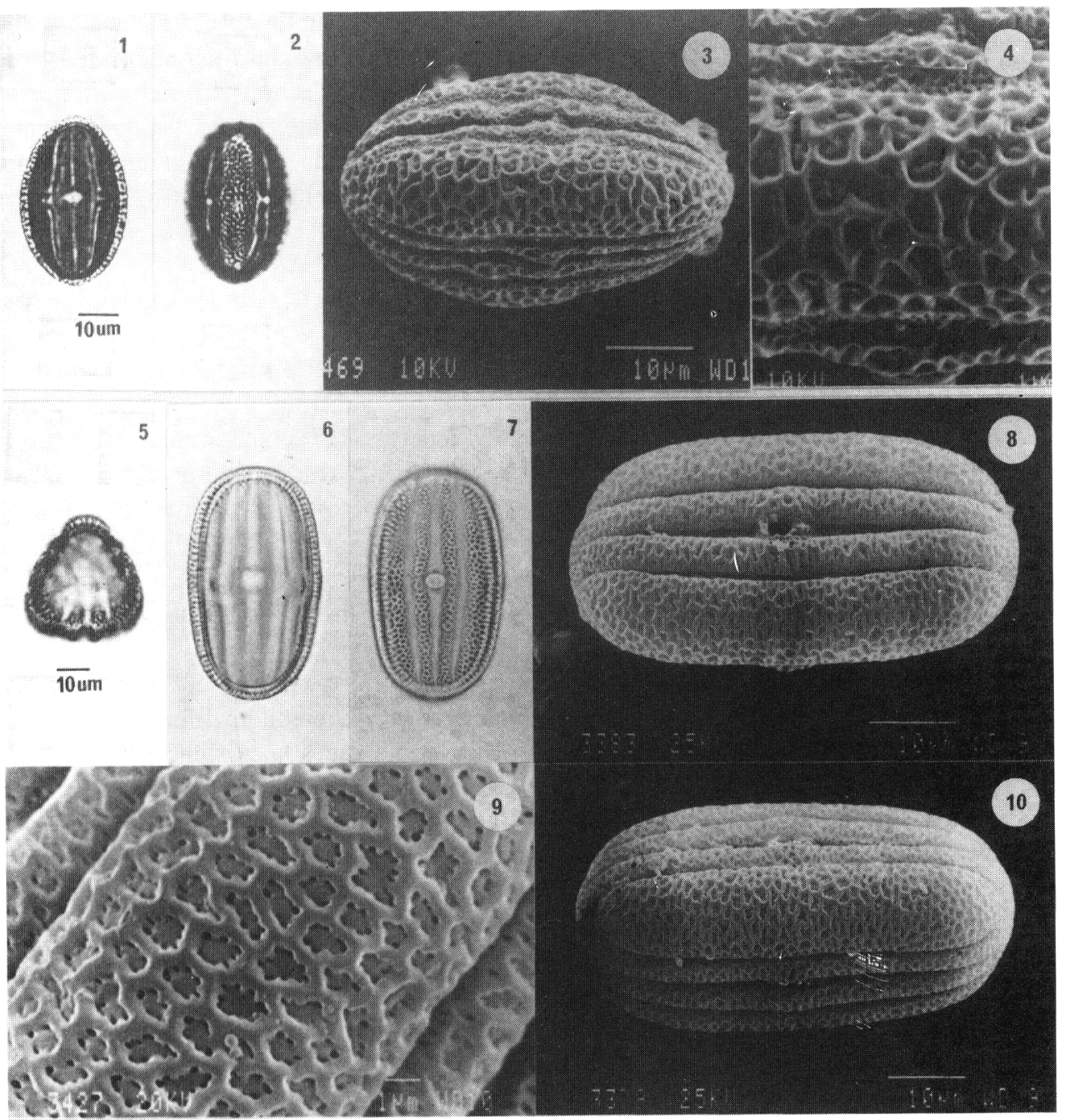

Figura 2. Hypoestes sanguinolenta: 1) v. e. y c.o.m. 2) v. e., superficie y abertura. 3) Aspecto general del polen, v.e. 4) Detalle de la superficie y aberturas. Jacobinia suberecta: 5) v.p. y c.o.e. 6) v.e. y c.o.m. 7) v.e., superficie y aberturas. 8) Aspecto general del polen, v.e. 9) Detalle de la superficie. 10) Aspecto general del polen, v.e.

bisimétrico. Elíptico en v.p. y c.o.e., elíptico obtuso en v.e. y c.o.m., de prolado a perprolado, siendo $\mathrm{P} / \mathrm{E}_{1}=1,65-2,04(1,84 \pm 0,11)$. Tamaño grande, con valores de $\mathrm{P}=80-93(85,84 \pm$ $3,17) \mu \mathrm{m}$, de $\mathrm{E}_{1}=41-52(46,76 \pm 2,52) \mu \mathrm{m}$ y de $E_{2}=22-29(25,33 \pm 2,51) \mu m$. Aberturas compuestas; ectoaberturas de tipo colpo, subter- minales, con los extremos agudos, de $25-30 \mu \mathrm{m}$ de longitud y la membrana apertural granulosa; endoaberturas de tipo poro, ligeramente lalongadas, a veces circulares, de $4 \times 4-5 \mu \mathrm{m}$. Exina de unos $3 \mu \mathrm{m}$ de grosor en los polos, con la sexina igual de gruesa que la nexina, y de 7 a $9 \mu \mathrm{m}$ en la mesocolpia, siendo entonces las 
sexina 2 veces más gruesa que la nexina debido al alargamiento de las columelas del infratéctum. Téctum parcial. Infratéctum columelado. Superficie finamente perforadoreticulada, apareciendo como elementos supretectales verrugas de hasta $3 \mu \mathrm{m}$ de diámetro regularmente dispuestas en los alrededores de los colpos (fig. 3).

Referencias: se desconocen.

Thunbergia alata Boj. (sur y oeste de Africa) Thunbergia laurifolia Lindl. (India)

Polen panto-espiraperturado. Apolar, asimétrico. Subcircular en corte óptico. Tamaño de mediano a grande, siendo $\mathrm{D}=40-51$ $(44,92 \pm 2,49) \mu \mathrm{m}$ en Th. alata y 61-75 $(68,66 \pm 3,97) \mu \mathrm{m}$ en $T h$. laurifolia. Abertura simple, helicoidalmente dispuesta por toda la superficie del grano de polen, a menudo de manera irregular y apareciendo a veces pequeños sulcos independientes; membrana apertural psilada. Exina de 3 a 3,5 $\mu \mathrm{m}$ de grosor en $T h$.

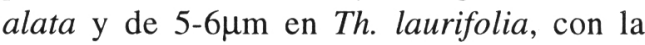
sexina de 2 a 3 veces más gruesa que la nexina. Téctum completo. Infratéctum sin columelas aparentes al M.O. Superficie escábrida en Th. alata y granulosa en Th. laurifolia (fig. 4).

Referencias: Erdtman (1952).

\section{VERBENACEAE}

\section{Citharexylum}

fruticosum

L. (Centroamérica).

Polen trizonocolporado. Isopolar, radiosimétrico. Triangular en v.p. y c.o.e., angulaperturado; de subcircular a ligeramente elíptico en v.e. y c.o.m. De suboblado a obladoesferoidal, siendo $\mathrm{P} / \mathrm{E}=0,86-1 \quad(0,92 \pm 0,04)$. Tamaño de pequeño a mediano, con valores de $\mathrm{P}=24-29(26,05 \pm 1,19) \mu \mathrm{m}$ y de $\mathrm{E}=26-31$ $(28,30 \pm 1,13) \mu \mathrm{m}$. Aberturas compuestas; ectoaberturas de tipo colpo, subterminales, de 10 a $14 \mu \mathrm{m}$ de longitud, con la membrana apertural escábrida; endoaberturas de tipo poro, lalongadas. Exina de aproximadamente $2 \mu \mathrm{m}$ de grosor, con la nexina ligeramente más gruesa que la sexina. Téctum perforado. Infratéctum con columelas muy cortas y poco patentes. Superficie psilada, con perforaciones dispersas e irregularmente dispuestas (fig. 4).

Referencias: se desconocen.

Duranta repens L. (sur de Estados Unidos a Brasil).

Polen trizonocolporado. Isopolar, radiosimétrico. Subtriangular en v.p. y c.o.e., angulaperturado; de subcircular a elíptico en v.e. y c.o.m. De oblado-esferoidal a subprolado, siendo $\mathrm{P} / \mathrm{E}=0,94-1,26(1,03 \pm 0,10)$. Tamaño mediano, con valores de $\mathrm{P}=31-30$ (34,5士 $2,17) \mu \mathrm{m}$ y de $\mathrm{E}=29-37(33,5 \pm 2,17) \mu \mathrm{m}$. Aberturas compuestas; ectoaberturas de tipo colpo, terminales, con la membrana apertural escábrida; endoaberturas de tipo poro, lalongadas y a menudo difusas, habiéndose observado a veces la existencia de vestíbulo. Exina de 2 a $2,5 \mu \mathrm{m}$ de grosor, con la sexina ligeramente más gruesa que la nexina. Téctum completo. Infratéctum con columelas cortas y poco patentes al M.O. Superficie psilado-perforada (fig. 4).

Referencias: se desconocen.

Lantana camara L. (América tropical).

Polen trizonocolporado. Isopolar, radiosimétrico. Subtriangular en v.p. y c.o.e., de subcircular a ligeramente elíptico en v.e. y c.o.m. De suboblado a subprolado, siendo P/ $\mathrm{E}=0,86-1,15(0,98 \pm 0,07)$. Tamaño mediano, con valores de $\mathrm{P}=28-38(31,54 \pm 2,98) \mu \mathrm{m}$ y de $\mathrm{E}=29-34(31,91 \pm 1,76) \mu \mathrm{m}$. Aberturas compuestas; ectoaberturas de tipo colpo, casi terminales, con la membrana apertural granulosa; endoaberturas de tipo poro, lalongadas, muy estrechas, con los extremos acuminados y a menudo constreñidos en la parte central. Exina de 2-2,5 $\mu \mathrm{m}$ de grosor en la mesocolpia, con la sexina aproximadamente igual de gruesa que la nexina, formando ésta última costillas a 


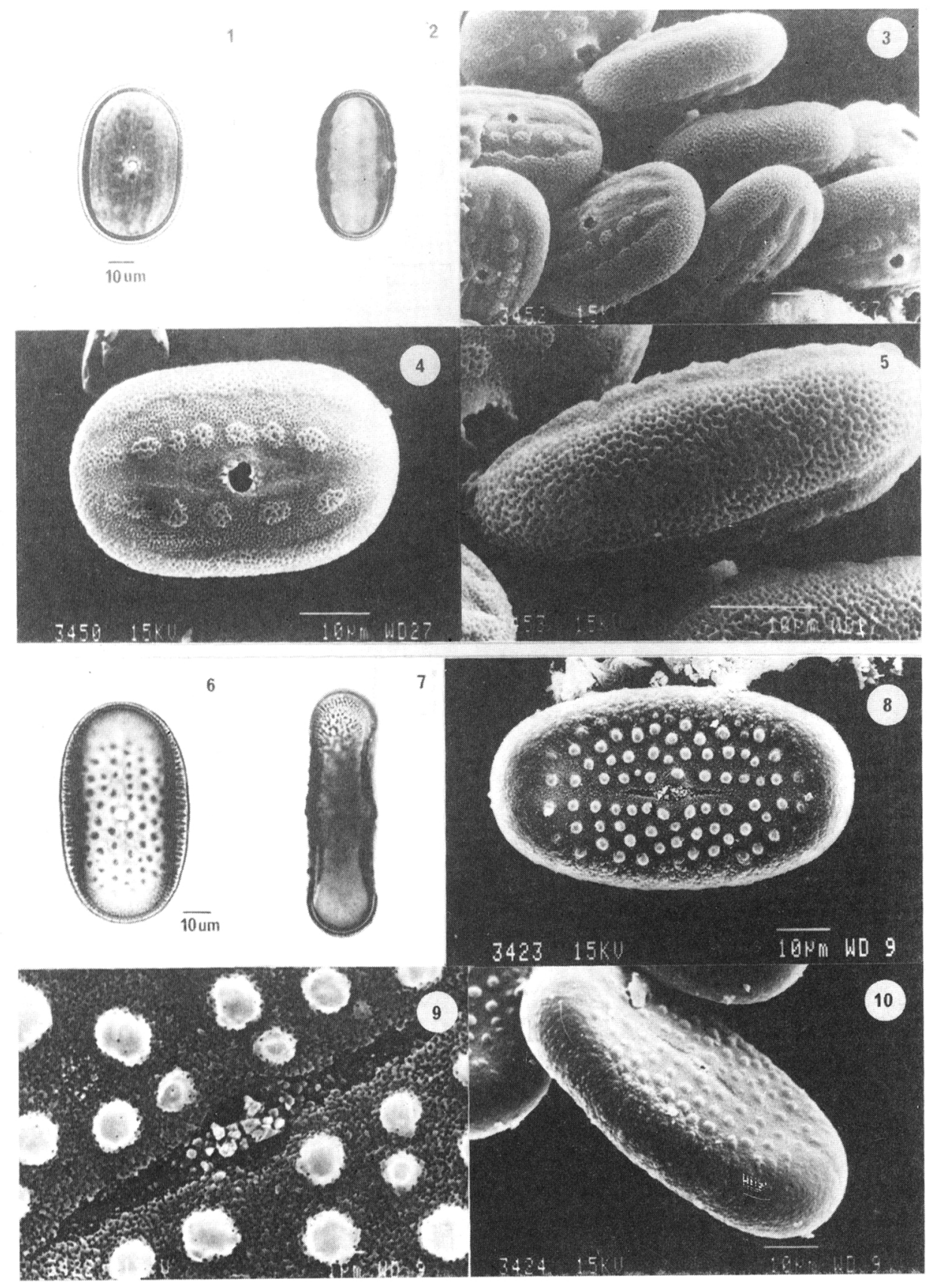

Figura 3. Justicia adhatoda: 1) v.e. y c.o.m. 2) v.e. y c.o.m. 3) Aspecto general de los granos de polen 4) Aspecto general del polen, v.e. 5) Aspecto general del polen v.e... Justicia carnea: 6) v.e... y c.o.m. 7) v.e. y c.o.m. 8) Aspecto general del polen, v.e. 9) Detalle de la superficie y abertura. 10) Aspecto general del polen, v.e. ${ }_{2}$. 
nivel de las endoaberturas. Téctum perforado. Infratéctum con columelas poco patentes. Superficie psilado-perforada (fig. 5).

Referencias: se desconocen.

Lippia triphylla (L'Herit) O. Ktze (Argentina y Chile).

Polen generalmente trizonocolporado, a veces tetrazonocolporado. Isopolar, radiosimétrico. De subcircular a subtriangular en v.p. y c.o.e., subcircular en v.e. y c.o.m. De suboblado a oblado-esferoidal, siendo $\mathrm{P} / \mathrm{E}=$ $0,81-1(0,91 \pm 0,05)$. Tamaño pequeño, con valores de $\mathrm{P}=16-20(17,80 \pm 1,08) \mu \mathrm{m}$ y de $\mathrm{E}=$ $18-21(19,66 \pm 1,04) \mu \mathrm{m}$. Aberturas compuestas; ectoaberturas de tipo colpo, subterminales, con los extremos dilatados y redondeados y la membrana apertural escábrida; endoaberturas de tipo poro, lalongadas y constreñidas en la parte central. Exina de aproximadamente $1 \mu \mathrm{m}$ de grosor, con la sexina unas 2 veces más gruesa que la nexina, formando ésta última costillas a nivel de las endoaberturas. Téctum completo, con algunas perforaciones. Infratéctum sin columelas aparentes al M.O. Superficie psilado-perforada (fig. 5).

Referencias: se desconocen.

\section{Verbena elegans H.B.K. (sur de México).}

Polen trizonocolporado. Isopolar, radiosimétrico. Subtriangular en v.p. y c.o.e., angulaperturado; de subcircular a ligeramente elíptico en v.e. y c.o.m. De oblado-esferoidal a subprolado, siendo $\mathrm{P} / \mathrm{E}=0,97-1,22$ $(1,13 \pm 0,07)$. Tamaño muy variable de mediano a grande, con valores de $\mathrm{P}=41-60$ $(47,8 \pm 5,69) \mu \mathrm{m}$ y de $\mathrm{E}=36-53(42,40 \pm 4,47) \mu \mathrm{m}$, habiéndose observado gran parte del material abortado. Aberturas compuestas; ectoaberturas de tipo colpo, casi terminales, con la membrana apertural escábrida; endoaberturas de tipo poro, lalongadas. Exina de aproximadamente $3 \mu \mathrm{m}$ de grosor en la mesocolpia, con la sexina mucho más gruesa que la nexina, formando ésta última costillas a nivel de las endoaberturas. Téctum perforado. Infratéctum columelado. Superficie psilado-perforada (fig. $5)$.

$$
\text { Referencias: Erdtman (1952). }
$$

Vitex agnus-castus L. (sur de Europa).

Polen trizonocolpado. Isopolar, radiosimétrico. Circular lobulado en v.p. y c.o.e., de subcircular a elíptico en v.e. y c.o.m. De oblado-esferoidal a subprolado, siendo P/ $\mathrm{E}=0,96-1,23(1,08 \pm 0,06)$. Tamaño de pequeño a mediano, con valores de $\mathrm{P}=20-30$ $(26,5 \pm 2,13) \mu \mathrm{m}$ y de $\mathrm{E}=20-27(24,55 \pm 1,76) \mu \mathrm{m}$. Aberturas simples, de tipo colpo, terminales, con la membrana apertural escábrida. Exina de aproximadamente $2 \mu \mathrm{m}$ de grosor en la mesocolpia, con la sexina igual o ligeramente más gruesa que la nexina. Téctum parcial. Infratéctum columelado. Superficie perforado-reticulada, con lúmenes irregulares de hasta $1 \mu \mathrm{m}$ de longitud y muros psilados (fig. 5).

Referencias: Pla Dalmau (1961), Luque y Candau (1987b).

\section{DISCUSIÓN}

Tras el estudio realizado, observamos la existencia de diversos tipos polínicos dentro de la familia Acanthaceae, como corresponde al carácter euripalinomorfo de la misma que ya había sido puesto de manifiesto por Erdtman (1952), apareciendo tipos trizonocolpados (Acanthus mollis), trizonocolporados (Eranthemum nervosum), heterocolpados (Hypoestes sanguinolenta y Jacobinia suberecta), bizonocolporados (Justicia adhatoda y $J$. carnea) y espiraperturados (Thunbergia alata y Th. laurifolia). En cuanto al tamaño, los granos de polen estudiados oscilan entre medianos y grandes y presentan la superficie muy diversamente ornamentada.

Dentro de la familia Verbenaceae, los táxones aquíincluidos presentan tipos polínicos más uniformes, oscilando entre el 

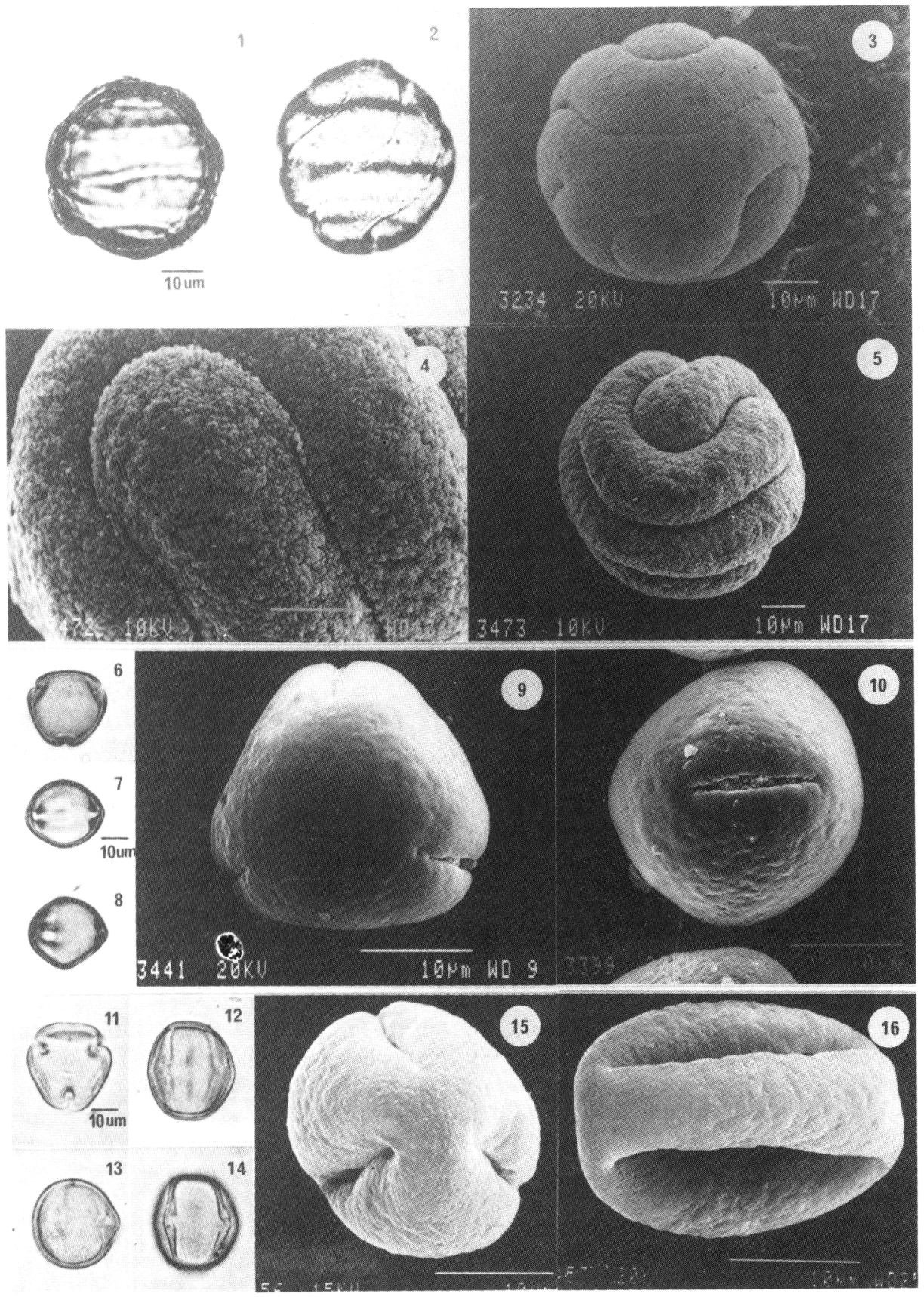

Figura 4. Thunbergia alata: 1) c.o. 2) Superficie. 3) Aspecto general del polen. Thunbergia laurifolia: 4) Detalle de la superficie y abertura. 5) Aspecto general del polen. Citharexylum fruticosum: 6) v.p. y c.o.e. 7) v.e. y c.o.m. 8) v.e., costillas. 9) Aspecto general del polen, v.p. 10) Aspecto general del polen, v.e. Duranta repens: 11) v.p. y c.o.e. 12-13) v.e. y c.o.m. 14) v.e., costillas.15) Aspecto general del polen, v.p. 16) Aspecto general del polen, v.e. 
trizonocolpado (Vitex agnus-castus) y trizonocolporado (Citharexylum fruticosum, Duranta repens, Lantana camara, Lippia triphylla y Verbena elegans), de tamaño mequeño o mediano y superficie psilado-perforada o perforado-reticulada.

En cuanto a las referencias que tenemos sobre trabajos anteriores, en general, los resultados obtenidos no difieren sustancialmente con las descripciones aportadas por otros autores, salvo diferencias poco significativas en lo que a tamaño se refiere, excepto en el caso de Vitex agnus-castus que fué descrito como trizonocolporado por Luque y Candau (1987).

\section{Anexo 1: Material examinado}

Acanthus mollis: Málaga: El Parque. 21-V-93 (MGC 17492).

Citharexylum fruticosum: Málaga: El Parque. 15VI-86 (MGC 17172).

Duranta repens: Málaga: Avda. de Andalucía. 8VII-85 (MGC 17395).

Eranthemum nervosum: Málaga: El Parque. 15VI-85 (MGC 16928).

Hypoestes sanguinolenta: Málaga: Avda. de la Aurora, terraza particular. 8-VIII-93 (MGC 17498).

Jacobinia suberecta: Málaga: Jardines de Picasso. 20-V-93 (MGC 17493).

Justicia adhatoda: Málaga: Ronda Intermedia. 26I-86 (MGC 16992).

Justicia carnea: Málaga: Finca de San José. 25-X88 (MGC 17393).

Lantana camara: Málaga: Jardines de Picasso. 20V-93 (MGC 17494).

Lippia triphylla: Málaga: Urb. El Limonar. 15VII-85 (MGC 17178).

Thunbergia alata: Málaga: Pasaje Horacio Lengo. 24-X-91 (MGC 17469).

Thunbergia laurifolia: Málaga: Fuengirola, Avda. de Mijas. 11-IX-93 (MGC 17499).

Verbena elegans: Málaga: Benalmádena-Costa. 9III-85 (MGC 16566).

Vitex agnus-castus: Málaga: jardines del Aeropuerto. 14-VIII-92 (MGC 17456).

\section{BIBLIOGRAFÍA}

BELMONTE, J., R. PEREZ-OBIOL y J.M. ROURE -1986- Claves para la determinación de los pólenes de las principales especies melíferas de la Península Ibérica. Orsis, 2: 27-54.

ERDTMAN, G.-1945 - Polen morphology and plant taxonomy. III. Morina L. with addition on morphological terminology. Svensk. Bot. Tidskr., 39: 187-191.

ERDTMAN, G. -1952- Pollen morphology and plant taxonomy. Angiosperms.(An introducction to palynology). Hafner Publ. Co., Stockholm.

ERDTMAN, G. -1960- The acetolysis method. Svensk. Bot. Tidskr., 54: 561-564.

FAEGRI, K. \& J. IVERSEN -1975- Texbook of pollen analysis. Munksgaard, Copenhagen.

HIDEUX, M. \& I.K. FERGUSON -1975Stereostructure de l'exine des Saxifragales: proposition d'une description logique et schematique. Bull. Soc. Bot. France 122: 57-67.

LUQUE, T. y P. CANDAU -1987a- Acanthaceae. En B. VALDÉS, M. J. DÍEZE I. FERNÁNDEZ (eds.). Atlas polínico de Andalucía Occidental: 310 Instituto de Desarrollo Regional de la Universidad de Sevilla y Excma. Diputación de Cádiz, Sevilla.

LUQUE, T. y P. CANDAU -1987b-Verbenaceae. En B. VALDÉS, M. J. DÍEZE I. FERNÁNDEZ (eds.). Atlas polínico de Andalucía Occidental: 281-283 Instituto de Desarrollo Regional de la Universidad de Sevilla y Excma. Diputación de Cádiz, Sevilla.

MATEU, I., A. AGUILELLA \& J.M. AZCARRAGA -1987- Pólenes del Jardín Botánico de Valencia. I. Anales Asoc. Palinol. Lengua Esp. 3: 73-90.

PLA DALMAU, J.M. -1961- Polen. Talleres gráficos D.P.C., Gerona.

REITSMA, T. -1969- Size modification of recent pollen grains under different treatments. Rev. Paeobot. Palynol., 9: 175-202.

REITSMA, T. -1970- Suggestions towards unification of descriptive terminology of Angiosperms pollen grains. Rev. Paleobot. Palinology 10: 39-60.

ROURE, J. -1985-Palinología Ibérica. Fam. 1 a 20. Cupressaceae a Betulaceae. Orsis, 1: 43-69.

TRIGO, M.M. -1989- Aportación al estudio palinológico de la flora ornamental de la ciudad de Málaga: Gimnospermas. Acta Bot. Malacitana 14: 238-244.

TRIGO, M.M. -1991- Contribución al estudio polínico de especies ornamentales: 

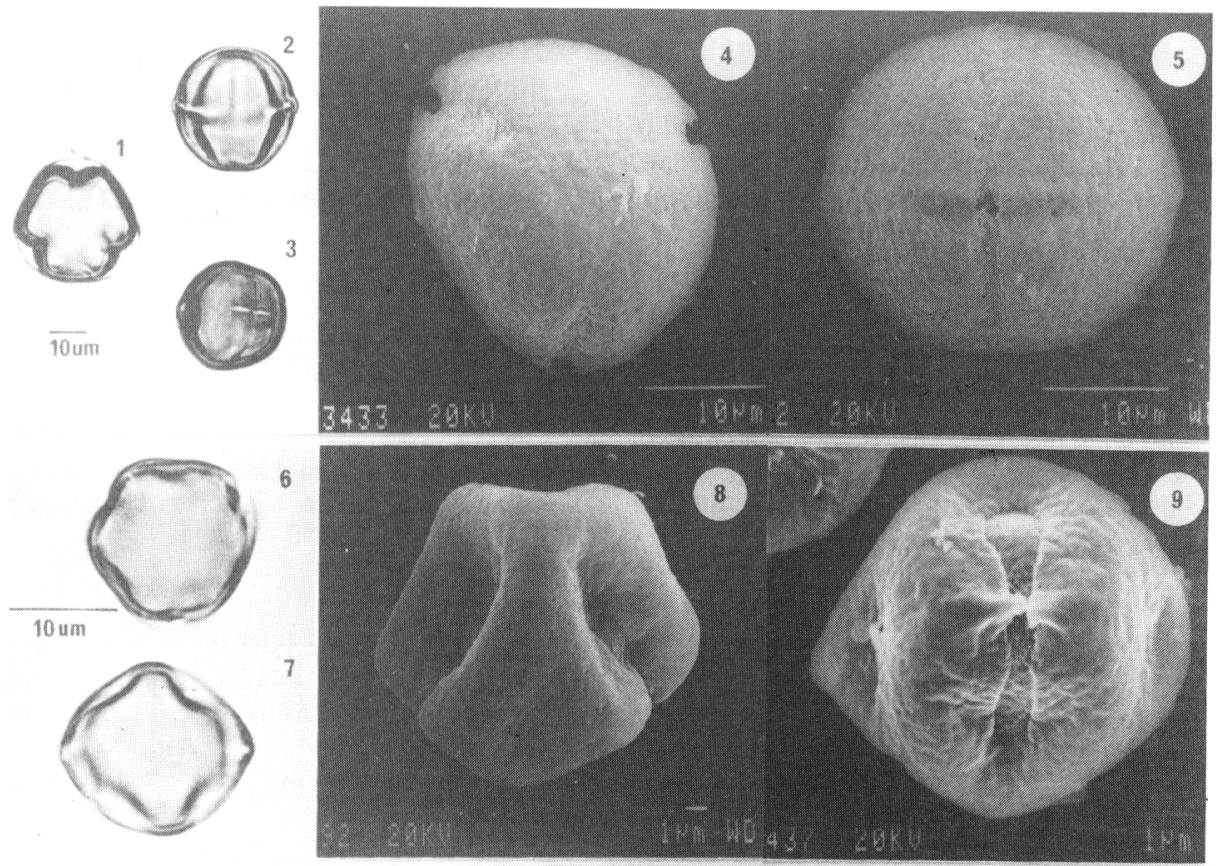

10
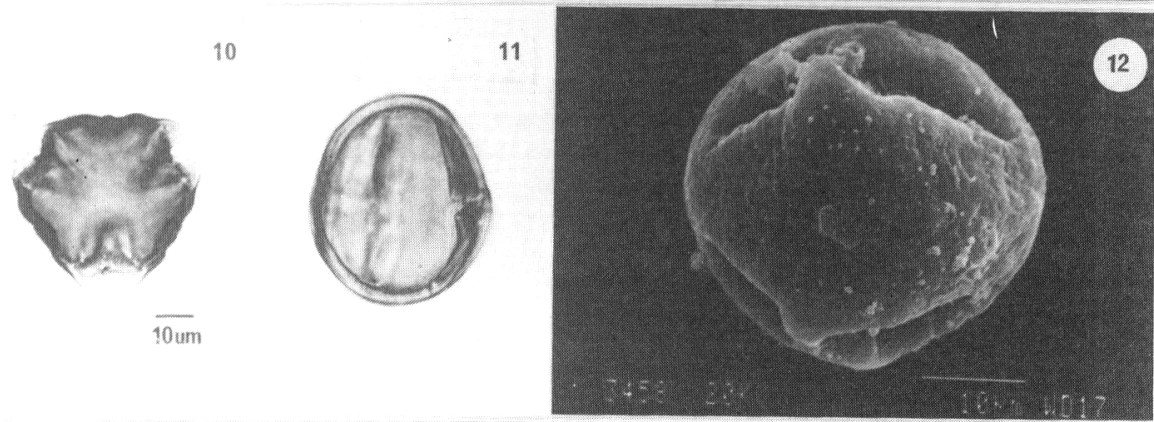

11

$\overline{10 u m}$

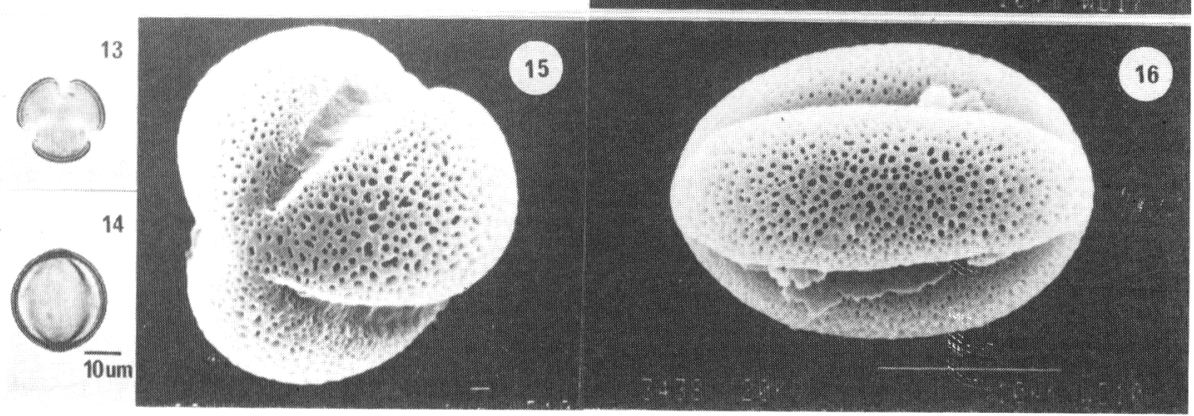

Figura 5. Lantana camara: 1) v.p. y c.o.e. 2) v.e. y c.o.m. 3) v.e., abertura. 4) Aspecto general del polen, v.p. 5) Aspecto general del polen, v.e. Lippia triphylla: 6) v.p. y c.o.e. 7) v.e. y c.o.m. 8) Aspecto general del polen, v.p. 9) Aspecto general del polen, v.e. Verbena elegans: 10) v.p. y c.o.e. 11) v.e. y c.o.m. 12) Aspecto general del polen, v.e. Vitex agnus-castus: 13) v.p. y c.o.e. 14) v.e. y c.o.m. 15) Aspecto general del polen, v.p. 16) Aspecto general del polen, v.e. 
Bignoniaceae. Acta Bot. Malacitana 16(2): 455466.

TRIGO, M.M. -1992a- Contribución al estudio polínico de especies ornamentales: Solanaceae, Convolvulaceae e Hydrophyllaceae. Acta Bot. Malacitana 17: 209-222.

TRIGO, M.M. -1992b- Flora ornamental y jardines de Málaga. Contribución al estudio de especies ornamentales con interés alergógeno. Universidad de Málaga. Colección Tesis Doctorales $\mathrm{n}^{\circ} 71$.

TRIGO, M.M. e I. GARCIA -1990- Morfología polínica de plantas ornamentales: Leguminosas. Acta Bot. Malacitana 15: 45-67.
VAN CAMPO, M. -1958- Palinologie africaine II. Bull. Inst. Franç. Afrique Noire, sér.A, Sci. Nat. 20: 753-760.

WODEHOUSE, R.P. -1935- Pollen grains. McGraw-Hill, New York. U.S.A.

Aceptado para su publicación en Junio de 1993

Dirección de la autora. Departamento de Biología Vegetal. Facultad de Ciencias. Universidad de Málaga. Ap. 59. 29080-Málaga. 